\title{
Household transmission of SARS, 2003
}

\author{
Samantha D. Wilson-Clark, Shelley L. Deeks, Effie Gournis, Karen Hay, Susan Bondy, Erin Kennedy, \\ Ian Johnson, Elizabeth Rea, Theodore Kuschak, Diane Green, Zahid Abbas, Brenda Guarda
}

\section{ABSTRACT}

Background: In the 2003 outbreak in Toronto (in Ontario, Canada) of severe acute respiratory syndrome (SARS), about $20 \%$ of cases resulted from household transmission. The purpose of our study was to determine characteristics associated with the transmission of SARS within households.

Methods: A retrospective cohort of SARS-affected households was studied to determine risk factors for household transmission. Questionnaires addressed characteristics of the index case, the household and behaviours among household members. Potential risk factors for secondary transmission of infection were assessed in regression models appropriate to the outcome (secondary cases) and nonindependence of household members.

Results: The 74 households that participated included 18 secondary cases and 158 uninfected household members in addition to the 74 index cases. The household secondary attack rate was $10.2 \%$ (95\% confidence interval [CI] $6.7 \%-$ $23.5 \%$ ). There was a linear association between the time the index patient spent at home after symptom onset and the secondary attack rate. Infected health care workers who were index cases had lower rates of household transmission.

Interpretation: SARS transmission in households is complex and increases with the length of time an ill person spends at home. Risk of transmission was lower when the index case was a health care worker. Rapid case identification is the public health measure most useful in minimizing exposure in the home.

CMAJ 2006;175(I0):1219-23

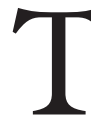
2003 outbreak of severe acute respiratory syndrome (SARS) was an example of how public health agencies and clinicians are able to provide guidance for prevention and control measures while determining modes of transmission, means of prevention and risk factors for infection.

We now know that SARS is an atypical pneumonia caused 2 by infection with the SARS coronavirus. ${ }^{1}$ The 2003 outbreak resulted in over 250 cases of SARS in Canada, mostly in Ontario in the Greater Toronto Area (GTA). ${ }^{2}$ Although most of the Ontario cases related to exposure in hospitals, a fifth of them resulted from household exposure. ${ }^{3}$

All GTA household members with a known exposure to a possible case of SARS were quarantined. The large range documented for secondary attack rates within households (0-100\%) suggested the presence of specific factors and behaviours that may increase risk of transmission in this setting (unpublished data, Toronto Public Health, 2003). Understanding these differences and identifying protective or riskincreasing behaviours may help prevent future household transmissions of emerging infectious diseases.

The objective of this study was 2-fold: to identify characteristics of the index case and household that increase the risk of transmission of SARS, and to identify individual behaviours both of the index case and household members that increase the risk of transmission within the context of households.

\section{Methods}

Cohort households were identified through surveillance databases from Toronto Public Health (TPH) and York Region Health Services Department (YR). These 2 adjacent health units accounted for $84 \%$ of SARS cases in Ontario: Toronto 228 , York Region 88, Ontario $375 .{ }^{4}$ TPH serves a large metropolitan area with a population of some 2.4 million people. YR serves another large, primarily urban area located just north of Toronto, with a population of about $730000 .{ }^{5}$ Eligible households were recruited into the study between May 25 and Oct. 31, 2003. The index case was defined as the first person in a household who met the SARS probable or suspect case definition as defined (May 29, 2003) by Health Canada, based on the date of onset of symptoms of SARS. ${ }^{6}$

Box 1: Definitions for a confirmed case of SARS

A person with both of 2 features:

- Early clinical presentation of SARS: fever $\left(>38^{\circ} \mathrm{C}\right)$ and a cough or breathing difficulty

- Radiographic evidence consistent with SARS; that is, of infiltrates consistent with pneumonia or RDS (details available at www.phac-aspc.gc.ca/sars-sras/\#note)

Or a deceased person with both of 2 features:

- A history of early clinical presentation of SARS: fever and a cough or difficulty breathing that resulted in death

- Autopsy findings consistent with SARS: evidence of pneumonia or RDS with no identifiable alternative cause

Note: SARS = severe acute respiratory syndrome, RDS = respiratory distress syndrome. 


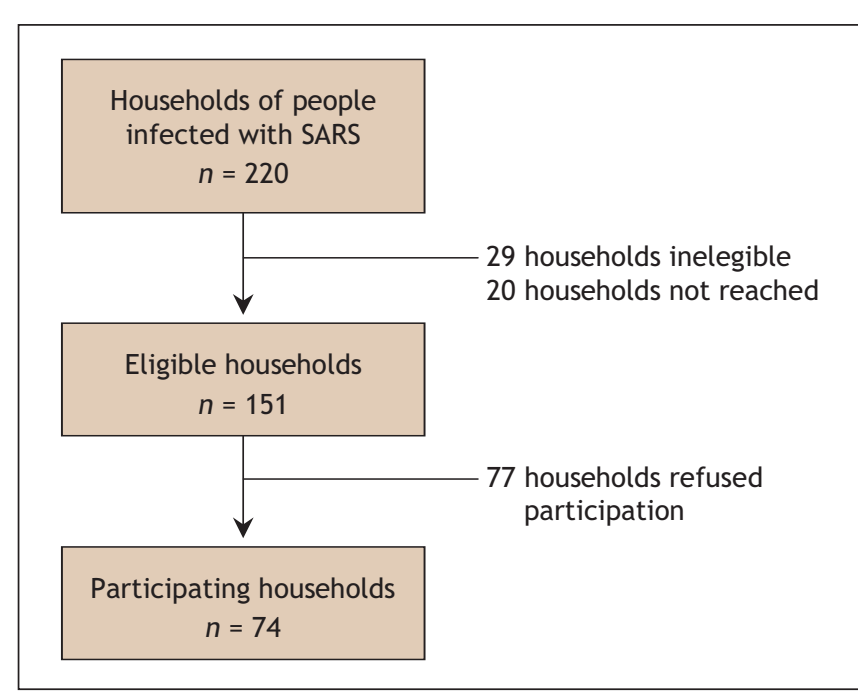

Fig 1: Participation rates, by household.

Households were eligible for inclusion if 1 person or more lived in the household with the index case during the period of interest: from the onset of the first SARS symptom to isolation of the patient. The case definitions we used for SARS are shown in Box 1. Households were excluded if the place of residence was an institution. Household members included everyone who lived in the residence of the index case or spent at least $20 \mathrm{~h} / \mathrm{wk}$ there during the index case's period of interest. The household member 15 years of age or older who was deemed (by available household members) to be the most knowledgeable about household activities was interviewed in person by a trained public-health nurse.

Characteristics of the index case and physical dwelling place, and the health status and behaviours of individual household members during the period of interest were documented (listed in full in an online Appendix, available at www .cmaj.ca/cgi/content/full/175/Io/1219/DC1). Behaviour questions included queries about hand hygiene (handwashing with soap and water or use of an alcohol-based hand sanitizer), use of preventive measures such as masks, and types and duration of household contact with the index case. Occupation and place of work were determined by self-report. Pastimes were defined to include leisure activities (watching TV, playing cards), engaging in conversation and other social interactions where people may be within 1 metre of each other.

Household transmission was defined as any additional case of SARS in the same household during the period of interest. Secondary SARS cases were defined as any SARS case with an onset of illness after that of the index case of the household (distinctions were not made between secondary, tertiary and other cases in the household). The secondary attack rate is the number of households (or household members) with secondary SARS cases divided by the total number of households (or household members) at risk.

Analysis followed the study design and was based on the assumption that transmission of SARS would be related to 3 potential interactions: the behaviours and illness pattern of the index case; the behaviours of all household members who were not the index case; and the characteristics of the household itself.

First analyses were performed to examine the influence of the household factors and behaviours of the index case on SARS transmission. The dependent variable was the number of secondary SARS cases in the household with the number of household contacts as the person-time denominator. The household was the first unit of analysis (i.e., What was the rate of transmission within this household?); a negative binomial regression model was used. Household members were the second unit of analysis (i.e., What is the risk of transmission to each person living in the household?). Poisson regression and related models were used to model the secondary attack rate in relation to covariates measured at both the household and individual household-member levels.

Interactions were observed between household factors, index case behaviours, and household member behaviours. To assess these interactions, a model adjusting for significant household factors and index case behaviours was used to estimate the risk of transmission to individual household members. Interaction terms were not tested on the following grounds: lack of a priori hypotheses of effect modification; low power to detect true effect modification; and to limit multiple comparisons and type II errors (false negatives).

The overall sample size of this analysis is small; the results should therefore be interpreted with some degree of caution. Limited data suppression was used to avoid presenting highly unreliable findings. Covariates were excluded if the groups being analyzed included fewer than 5 unique individuals or

Table 1: Characteristics of index cases of SARS, secondary cases and household members uninfected by SARS in 74 affected households

\begin{tabular}{|c|c|c|c|c|}
\hline \multirow[b]{2}{*}{ Descriptor } & \multicolumn{4}{|c|}{ Number of people $(\%)$, unless otherwise indicated } \\
\hline & $\begin{array}{l}\text { Index/source cases } \\
\qquad n=74\end{array}$ & $\begin{array}{c}\text { Secondary cases } \\
n=18\end{array}$ & $\begin{array}{l}\text { Household members } \\
\qquad n=158\end{array}$ & Overall \\
\hline Age, median, yr & 43.5 & 34.5 & 34 & 37 \\
\hline Age range, yr & $1.5-77$ & $6-78$ & $1-79$ & $1-79$ \\
\hline Sex: female & $50(67.6)$ & $10(55.6)$ & $72(45.6)$ & $132(52.8)$ \\
\hline Occupation: health care worker & $50(67.6)$ & $1(5.6)$ & $10(6.3)$ & $61(24.4)$ \\
\hline Health status: underlying/pre-existing medical conditions & $42(56.8)$ & 7 (38.9) & $71(44.9)$ & $120(48.0)$ \\
\hline Data missing & $3(4.1)$ & 0 & $6(3.8)$ & $9(3.6)$ \\
\hline
\end{tabular}


households. Modelling was carried out at 3 levels: household, individual household members, and combined variables to measure household risk and contact at risk. In each case, univariate models were modelled first. Multivariable models included only risk factors reaching $p<0.1$ on their own. Models were reduced to exclude nonsignificant predictor variables. Extensive assessment of confounding effects of one variable on another was conducted with use of models of subsets of exposure variables that were intercorrelated.

Ethics approval was obtained from the Health Canada Research Ethics Board, TPH and YR.

\section{Results}

The study cohort included 317 cases of SARS, occupying 220 households. Of the $18 \mathrm{o}$ households $(81.8 \%$ ) successfully contacted, $151(83.9 \%)$ were eligible, and $74(49.0 \%)$ agreed to participate (Fig. 1). Data were obtained for 250 people: 1 index case per household and 176 in-household contacts (of whom 18 [10.2\%] became infected).

Of the 74 index cases, two-thirds were female and the same proportion worked in health care (Table 1 ). The 18 secondary cases belonged to 10 households. Overall, the household transmission rate was $13.5 \%$ (95\% confidence interval [CI] $6.2 \%-15.7 \%$ ); the secondary attack rate among all household members, $10.2 \%$ (95\% CI $6.7 \%-23.5 \%$ ). Secondary cases were comparable with the 158 noninfected house-

Table 2: Significant associations between SARS index-case characteristics and secondary attack rates, by unadjusted regression models* predicting the secondary attack rate $\dagger$

\begin{tabular}{|c|c|c|}
\hline \multirow[b]{2}{*}{$\begin{array}{l}\text { Patient characteristic, } \\
\text { index case }\end{array}$} & \multicolumn{2}{|c|}{ Unit of analysis; relative risk $(95 \% \mathrm{Cl})$} \\
\hline & $\begin{array}{l}\text { Household } \neq \\
\quad n=74\end{array}$ & $\begin{array}{l}\text { Individual§ } \\
n=176\end{array}$ \\
\hline Health care worker & $0.15(0.03-0.73)$ & $0.33(0.10-1.06)$ \\
\hline Age $\geq 60 \mathrm{yr}$ & $1.91(0.18-19.76)$ & $1.02(1.00-1.04)$ \\
\hline $\begin{array}{l}\text { Potential exposure period, } \\
\text { continuous? }\end{array}$ & $1.39(1.11-1.74)$ & $1.27(1.20-1.36)$ \\
\hline \multicolumn{3}{|l|}{ Exposure-period categories } \\
\hline $0-2-d$ and 3-4-d periods ${ }^{* *}$ & 1.0 & 1.0 \\
\hline $5-7 d$ & $3.92(0.58-26.50)$ & $5.78(1.51-22.13)$ \\
\hline $8-16 d$ & $11.14(1.32-93.89)$ & $7.03(1.65-30.00)$ \\
\hline \multicolumn{3}{|l|}{ Household behaviours } \\
\hline Shared kitchen towels & $9.70(1.26-74.36)$ & $7.89(1.78-34.91)$ \\
\hline Shared bathroom cup & $9.12(1.62-51.38)$ & $6.48(2.17-19.38)$ \\
\hline
\end{tabular}

Note: $\mathrm{Cl}=$ confidence interval

*Negative binomial regression models, unadjusted, each with a single predictor variable. The independent variable is the (binary) transmission status for the person exposed to SARS via the index case in that household. tIncidence of secondary transmission of SARS among people identified as household contacts of index SARS cases.

$\neq$ The independent variable is the count of secondary transmission cases per household, with the no. of at-risk household contacts set as a person-time offset covariate.

SIndividuals are clustered in 74 households with an index SARS case. The independent variable is the (binary) transmission status for individual household contacts of the 74 index SARS cases (total $n=176$ people at risk). IDays from symptom onset to hospital admission.

** Reference period for analysis by exposure-period category.

hold members in age, sex proportion and percentage of index cases who were health care workers.

\section{Risk factors in people who were index cases}

The number of days the index case spent at home after the onset of SARS symptoms was a significant risk factor for secondary household transmission (Table 2), with a linear relationship in which longer exposure increased the risk of transmission per day exposed (relative risk [RR] 1.4, 95\% CI 1.11.7). Households of an index case who was a health care worker had a secondary attack rate $85 \%$ lower than that of the other households. The health status (i.e., existence or absence of an underlying illness) of the index case and her or his severity of SARS symptoms did not predict transmission risk.

Although several behavioural risk factors were examined, only 2 index-case behaviours predicted risk of secondary transmission: households in which the index case shared kitchen towels (RR 7.9, 95\% CI 1.8-34.9) or bathroom cups (RR 6.5, 95\% CI 2.2-19.4; Table 2). The risk factors associated with index-case characteristics (Table 3) were not independent of one another. When the index case worked in health care, he or she was less likely to have had a long period between onset of symptoms and isolation, and less likely to have shared kitchen towels or bathroom cups in the home. Number of days from onset to hospital admission was also strongly associated with the likelihood of reported towel sharing. Time from symptom onset to hospital admission remained statistically significant after considering these risk factors together. No association was found between specific symptoms of the index case (e.g., diarrhea) and increased transmission in the household.

\section{Risk factors in household members}

Univariate analyses examined hand hygiene practices, care provided to the index case and use of preventive measures (Table 4). After adjusting for the factors determined as significant for the index case, sharing of kitchen towels (RR 5.7,

Table 3: Significant associations between SARS index-case characteristics and secondary attack rates, by negative binomial regression models* predicting secondary attack rate $\dagger$ among 176 household contacts in 74 households with an index SARS case

Characteristic or behaviour of index case

Health care worker

Period, in days, from symptom onset to hospital admission, continuous

Shared kitchen towels

Shared bathroom cup

*Individual risks clustered in households as the unit of analysis.

†ncidence of secondary transmission of SARS among people identified as household contacts of index SARS cases. Multivariable models were adjusted simultaneously for 4 household-level covariates found to be associated with the secondary attack rate.

flndependent variable is (binary) transmission status for individual household contacts of index SARS patients. 
95\% CI 2.0-16.6), not always washing hands after caring for ill person (RR 3.5, 95\% CI 1.1-10.9) and not always washing hands after changing a diaper (RR 3.9, 95\% CI 1.3-12.1) were associated with an increased risk of SARS transmission.

Practices such as assisting the index case with toilet use, washing, walking and adjusting pillows for were significantly associated with an increased risk of SARS transmission. After adjustment for key risk factors associated with index-case characteristics (i.e., significant variables from Table 2), however, these variables became nonsignificant. Two of the variables were protective: engaging in household pastimes with ill person (RR 0.1, 95\% CI 0.02-0.6) and spending time in close contact with the index case (RR 0.2, 95\% CI 0.05-0.6). The number of masks worn by the household member, however, was a risk factor in the adjusted model (RR 1.2, 95\% CI 1.01.3): use of additional masks each day was associated with a $17 \%$ increase in the risk of secondary cases. Household factors such as forced-air heating, presence of pets and number of members in the household were unassociated with an increased risk of transmission.

\section{Interpretation}

Our study found that household members are at risk for SARS and that this risk increases with the amount of time spent by the index case in the household while ill. Risk of transmission was lower when the occupation of the index case was that of a health care worker. The primary risk factors for transmission fell into 3 areas: the duration of time spent in the home after the index case became ill, poor hand hygiene practices and poor use of respiratory protective measures. Two Asian studies have also examined transmission of SARS in households. ${ }^{7,8}$ The secondary attack rate for households in our study, $13.5 \%$, is comparable with the published study results from Singapore $(12.3 \%)^{7}$ and Hong Kong (14.9\%). ${ }^{8} \mathrm{Al}-$ though the household-member secondary-attack rate we found, $10.2 \%$, was higher than those from Singapore $(6.2 \%)^{7}$ and Hong Kong ( $8 \%),{ }^{8}$ the fact that the confidence intervals overlap indicates that the results are comparable. One of the most important factors for household transmission was duration of exposure in the home. We found a linear association between the number of days the ill index case remained at home and the secondary attack rate; similar results were seen in Hong Kong. ${ }^{8}$ Households where the index case worked in health care had a significantly lower secondary-attack rate than other households, an association also noted in the other studies. ${ }^{7,8}$ Possible explanations for the lower attack rates in such households include increased familiarity with the use of personal protective equipment and better infection-control practices in the home.

Factors related to hand hygiene were associated with increased risk of transmission. This finding speaks to the need for good handwashing practices in the home and recognition of potential vehicles of transmission, such as kitchen towels. Providing personal care was associated with increased transmission, consistent with droplet transmission of the SARS coronavirus. Interestingly, we found in the adjusted model that engagement in pastimes with the index case and time spent within 1 metre of that person were both factors that were protective against SARS infection. These hypotheses

Table 4: Associations between household-member behaviours and secondary attack rates, by regression models* predicting secondary attack ratet among 176 household contacts in 74 households with an index case of SARS

Relative risk ( $95 \%$ confidence interval)

\begin{tabular}{|c|c|c|}
\hline \multirow[b]{2}{*}{ Behaviour of household members } & \\
\hline & $\begin{array}{l}\text { Unadjusted regression for } \\
\text { significant risk factors for each } \\
\text { household member at risk }\end{array}$ & $\begin{array}{c}\text { Reduced multiple-predictor regression } \\
\text { models } \neq \text { showing associations between } \\
\text { individual-level risk factors and transmission }\end{array}$ \\
\hline \multicolumn{3}{|l|}{ Failure to wash hands } \\
\hline After caring for household member who was ill & $3.98(1.30-12.17)$ & $3.46(1.10-10.92)$ \\
\hline After changing diapers & $4.10(0.83-20.23)$ & $3.94(1.28-12.10)$ \\
\hline Shared kitchen towel & $5.25(1.51-18.28)$ & $5.71(1.96-16.63)$ \\
\hline \multicolumn{3}{|l|}{ Intimate contact with ill person } \\
\hline Engaged in household pastimes & $0.11(0.02-0.74)$ & $0.10(0.02-0.61)$ \\
\hline Time in close contact & $0.22(0.06-0.72)$ & $0.18(0.05-0.65)$ \\
\hline Assisted with toilet & $5.68(1.98-16.29)$ & - \\
\hline Assisted with washing & $4.49(1.47-13.66)$ & - \\
\hline Assisted with walking & $5.23(1.76-15.55)$ & - \\
\hline Adjusted pillows & $3.55(1.26-10.04)$ & - \\
\hline Respiratory protection, by no. of masks worn§ & $1.15(0.98-1.36)$ & $1.17(1.01-1.31)$ \\
\hline
\end{tabular}

*Negative binomial regression models. Independent variable is (binary) transmission status for individual household contacts of index SARS cases. †Incidence of secondary transmission of SARS among household contacts of index SARS cases.

†Reduced multivariable models include predictor variables measured at the level of household member at risk that are significantly associated with the outcome in multivariable models, or that serve as important confounders in other relationships shown. Models are also adjusted for most important confounders measured at the level of the entire household: whether the index case was a health care worker, and number of days from onset of symptoms to hospital admission. (Multiple regression was not performed on variables found to be nonsignificant by unadjusted analysis.)

$\S 0-4$ masks treated as a continuous number; $>4$ masks, fixed at 4. 
may explain this finding: Index cases who were able to engage in pastimes may have been less ill. Cases requiring assistance with toilet use may have been more ill and required closer or prolonged contact with their household members. Moreover, the SARS coronavirus is shed in feces; $;^{9,10}$ those who assisted in toilet use may have had an increased fecal exposure to the virus.

The "number of masks used each day" by household contacts was the only behavioural factor related to respiratory protection that was positively associated with household transmission of SARS. The transmission rate increased with the number of masks worn each day, independent of the maximum duration of exposure to the index case. This may have been due to droplet transmission from improper removal of masks, or a measure of severity of the SARS illness not captured in our analysis. Household size, dwelling type, mode of heating and number of household members were unassociated with secondary transmission. However, these findings need to be interpreted in the Canadian context, where less than $1 \%$ of homes are considered crowded (i.e., have more than one person per room). ${ }^{11}$ The presence of pets or rodents was also unassociated with secondary transmission.

Several important limitations of this study should be noted. First, the questionnaire was extensive, because knowledge about SARS transmission was limited. Risk factors were defined inclusively. This raises concerns about type I errors (finding a statistically significant association where one does not exist). Actual probabilities have been explicitly listed so that readers can make their own judgment. People designated as secondary cases might have acquired their infection outside the home; nevertheless, we believe the consistency of our findings with other studies supports the model of household transmission. Our results can be generalized to other households in urban communities in countries that have similar demographics and access to health care.

SARS provided a unique opportunity to study transmission of an infectious disease within households at a time when little was known about this disease. This study's findings indicate that new control measures should include rapid medical assessment and removal of the case from the home, as well as increased awareness of the importance of protective measures after symptom onset. Public health measures such as home isolation should be aimed at minimizing such risk factors when addressing household transmission of serious infections spread through droplet transmission.

Samantha Wilson-Clark is an Epidemiologist at Waterloo Region Public Health in Ontario, Canada. As a Federal Field Epidemiologist, she worked with the Pubic Health Agency of Canada's Immunization and Respiratory Infections Division. She was the first field epidemiologist sent to investigate a cluster of SARS cases in 2003 among health care workers in a Toronto hospital. She is a currently a member of the Ontario Ministry of Health and Long-Term Care's Emergency Management Unit's Scientific Advisory Council. Samantha completed her degree in Nursing in 1998 and in Epidemiology in 2002.
This article has been peer reviewed.

From the Canadian Field Epidemiology Program, Ottawa Ont. (WilsonClark, Abbas), the Division of Immunization and Respiratory Infections in Ottawa, Ont. (Deeks, Wilson-Clark), and the National Microbiology Laboratory in Winnipeg, Man. (Kuschak), of the Public Health Agency of Canada; and City of Toronto Public Health (Gournis, Kennedy, Rea, Green), York Region Health Services (Hay, Guarda) and University of Toronto (Bondy, Johnson), Toronto, Ont.

\section{Competing interests: None declared.}

Contributors: Samantha Wilson-Clark, Shelley Deeks, Susan Bondy and Effie Gournis participated in study conception and design, data analysis and interpretation, and the drafting of the manuscript. Ian Johnson, Theodore Kuschak and Zahid Abbas also contributed to the study concept and design. Ian Johnson and Diane Green were involved in the analysis and interpretation of data. Effie Gournis, Karen Hay, Erin Kennedy, Elizabeth Rea and Brenda Guarda acquired the data and contributed to its interpretation. All coauthors revised the manuscript critically and approved the version to be published.

Acknowledgements: We acknowledge the contributions of Susan G. Squires, Sandy Isaacs and Theresa Tam, Health Canada; Steven Jones, Daryl Dick and Lisa Fernando, National Microbiology Laboratory; Vartouhi Jazmaji and Lee Vernich, Research Services Unit, Public Health Sciences, University of Toronto; Barbara Yaffe, Bonnie Henry, Rebecca Stuart, Lucia Bresolin, Sukaina Champsi, Leah Choy, Mary Ann Gatbonton, Yvette Geridis, Kerry Gordon, Shauna Grant, Bernadette Kint, Linh Nguyen, Claudine D'Souza, Wayne Fletcher and Jane Griffiths, Toronto Public Health; and Pam Cornelius, Jennifer Santoianni, Nancy Hogue, Etta Li, Susan Ludwig, Susan O'Gorman, Christina Tietz, Diane Bladek-Willett, Bill Mindell and Hanif Kassam, York Region.

\section{REFERENCES}

1. Department of Communicable Disease Surveillance and Response. World Health Organization. Consensus document on the epidemiology of severe acute respiratory syndrome (SARS). May 2003. Available: www.who.int/csr/sars/en/WHOconsensus .pdf (accessed 2006 Sept 11).

2. World Health Organization. Summary of probable SARS cases with onset of illness from 1 November 2002 to 31 July 2003. Available: www.who.int/csr/sars/country Itable2004_04_21/en (accessed 2006 Sept 11).

3. Public Health Agency of Canada. Epidemic curve of a SARS outbreak in Canada, February 23 to 2 July 2003. Available: www.phac-aspc.gc.ca/sars-sras/pdf-ec/ec _20030808.pdf (accessed 2006 Sept 11).

4. Ontario Ministry of Health and Long-Term Care. Diseases: severe acute respiratory syndrome (SARS). Available: www.health.gov.on.ca/english/public/pub/disease /sars.html (accessed 2006 Sept 11).

5. Statistics Canada. 2001 community profiles. Available: www12.statcan.ca/english /profilo1/CP01/Index.cfm?Lang=E (accessed 2006 Sept 11).

6. Public Health Agency of Canada. National case definitions for the SARS outbreak period, 2003. Available: www.phac-aspc.gc.ca/sars-sras/sarscasedef_e.html (accessed 2006 Sept 11).

7. Goh DL, Lee BW, Chia KS, et al. Secondary household transmission of SARS, Singapore. Emerg Infect Dis 2004;10:232-4

8. Lau JTF, Kim JH, Wong E, et al. Probable secondary infections in households of SARS patients in Hong Kong. Emerg Infect Dis 2004;10:235-43.

9. Cheng PK, Wong DA, Tong LK, et al. Viral shedding patterns of coronavirus in patients with probable severe acute respiratory syndrome. Lancet 2004;363:1699-700.

10. Leung WK, To KF, Chan PK, et al. Enteric involvement of severe acute respiratory syndrome-associated coronavirus infection. Gastroenterology 2003;125:1011-7.

11. Silver C, Van Diepen R. Statistics Canada. Canadian social trends, February 6 , 2004. Available: www.statcan.ca/english/ads/11-008-XIE/housinge.html (accessed 2006 Sept 12).

Correspondence to: Samantha Wilson-Clark, Region of Waterloo Public Health, 99 Regina St. South, 3rd Floor, Waterloo ON $\mathrm{N}_{2} \mathrm{~J}_{4} \mathrm{~V}_{3}$; fax 519 883-2241; wsamanth@region.waterloo.on.ca 